\title{
NAVIGATION IN AN AUDIO-ONLY FIRST PERSON ADVENTURE GAME
}

\author{
Adrian Jäger \\ Department of Media Production \\ OWL University of Applied Sciences \\ Liebigstrae 87, \\ 32657 Lemgo, Germany \\ jaegerva@gmail.com
}

\author{
Aristotelis Hadjakos \\ Center of Music and Film Informatics \\ Detmold University of Music \\ OWL University of Applied Sciences \\ Hornsche Str. 44, 32756 Detmold, Germany \\ hadjakos@hfm-detmold.de
}

\begin{abstract}
Navigation in audio-only first person adventure games is challenging since the user has to rely exclusively on his or her sense of hearing to localize game objects and navigate in the virtual world. In this paper we report on observations that we made during the iterative design process for such a game and the results of the final evaluation. In particular we argue to provide a sufficient number of unique sound sources since players do not use a mental map of the virtual place for navigating but instead move from sound source to sound source in a more linear fashion.
\end{abstract}

\section{INTRODUCTION}

Since the invention of the first computer games about 50 years ago, significant progress has been made. Numerous milestones were achieved from the early text-based games and sprite graphics, to the simulation of three-dimensional worlds, massively multiplayer online games and the development of virtual reality. Also for sound and music, a game developer has many options today, including recorded music and sound samples, synthesized sounds, generated music and spatial audio. Sound and music are used in almost all games today, e.g., to create a desired emotional response with sound effects and background music or to develop the story with narration. However, the human-machine interaction in mainstream games is mainly visual: The user interacts with a virtual world that is mainly graphically represented and performs actions on visual elements. While the user's actions relate to vision, the feedback is often both visual and auditive. This makes it much more difficult but not impossible to play a game without visual feedback (as we know from visually impaired people who play mainstream games with some success [1]).

In audio games, the interaction is mainly auditive. The user interacts with an auditive virtual world without (or with very little) visual feedback. Audio games have a unique aesthetic quality and can stimulate the player's imagination. Thus a good audio game should normally be equally interesting for visually impaired and unimpaired people. Audio games are of course a niche genre.

A particular challenge for audio-only first person adventure games is navigation in the virtual world. Since there are no visual cues, the player has to rely on his or her hearing skills to localize

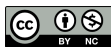

This work is licensed under Creative Commons Attribution Non Commercial 4.0 International License. The full terms of the License are available at http://creativecommons.org/licenses/by-nc/4.0 sounding objects and navigate in the world. While there are some studies on navigating in virtual environments (see Section 2.2), this paper examines navigation in the context of audio-only gaming. We report our observations that we gained during the iterative design of the game (see Section 5). We then discuss the results that we obtained in an evaluation of the game (see Sections 6 and 7).

\section{RELATED WORK}

\subsection{Audio games}

Wolf categorizes graphical computer games by genre [2]. He distinguishes the games based on the primary interaction characteristics offered to the player, the goals to be achieved, the type of control, and the player's avatar. On the basis of these criteria he describes 42 different genres, some of which also apply to audio games.

\subsubsection{Text Adventures}

In text adventures, the narration of the story is done by a computer voice or a recorded human voice without visual feedback. The user can select an action from a list of options. By doing this, a branch in the course of the story is chosen, which can have short- or longterm effect. An example of an audio text adventure is "Descent into Madness."1

\subsubsection{Grid games}

While many games are played in the first person perspective, some games limit the freedom of movement of the player to a fixed grid consisting of squares of unit length while the game world can still be explored relatively freely. The grid functions as a support and makes sure that game-relevant objects can not be so easily missed.

An example is "3D Snake," an adaptation of the famous "Snake" game. Using the arrow keys, the player navigates over a two-dimensional plane. When approaching the limits of the playing ground, this is announced by a characteristic sound that gets louder and louder. The fruits to be collected are recognizable by their own sound, which (again) gets louder as the distance between the player and the fruit decreases.

\footnotetext{
${ }^{1}$ Descent into Madness: http: / / www . cs. unc.edu/Research/ assist/et/2005/SoundsLikeFun.html, last access: Feb. 2017.

${ }^{2} 3 \mathrm{D}$ Snake: http://usagamesinteractive.com/ freeware. php, last access: Feb. 2017.
} 


\subsubsection{First-person adventures}

First-person adventures offer greater freedom than grid games: The player can move freely in the virtual world independently of a grid. Neither the length of the step, nor the angles of a rotation to the right or left are perceived as being discrete. The game principle calls for a realistic, auditive virtual reality.

The game "Slender: Lost Vision"3 can serve as a suitable example. As the name implies, this is an audio version of the game "Slender - The Eight Pages". ${ }^{4}$ The main character is a girl, who walks into a nightly forest out of curiosity. The girl has to collect eight pieces of paper attached to different trees without being caught by the Slenderman. The paper notes are rustling in the wind and can be heard by the player. With the right mouse button, an audio compass can be triggered that generates a sound located in the northern direction to make the orientation somewhat easier. But also the birds, which sit in the trees, help with the orientation. The game creates a lot of excitement through a buzzing, pulsating background music and sudden, frightening, loud noises that indicate the presence of the Slenderman.

\subsubsection{Side scrollers}

Similar to their visual counterparts, audio side scroller games run one-dimensionally from left to right. Obstacles that need to be skipped or repelled, and any auxiliary items that can be collected are announced by audio signals. These signals are usually learned beforehand in a short tutorial.

"Adventure at C",5 works this way.The player is a programmer whose hard drive is infected with viruses. To solve this problem, he goes on a journey through his hard drive and encounters viruses, which he has to fight, holes that he must skip and other obstacles. A common challenge in audio side scrollers is limited or missing feedback, which can make it hard to learn from past runs and perform the right corrective action.

\subsubsection{Rhythm and skill games}

Most of the categories listed above have in common that they either follow a story or evoke a space, a world. This is different in this category: the games require a mostly rhythmic interaction in which the player has to press a button or perform a specific movement. In some cases, the timing of the interaction is not relevant but the order of the pressed buttons, wherein the time intervals therebetween may be arbitrary.

An example of such a game is "Simon". ${ }^{6}$ The game consists of a device with four buttons, which produce different sounds. The built-in computer first plays a series of few sounds, which must then be reproduced by the player on the keys. If successful, the melody is extended by one note at the end and the player must play the now expanded melody from beginning to end. This scheme continues until the sound series is complete or the player makes a mistake.

\footnotetext{
${ }^{3}$ Slender - Lost Vision: http://dragonapps.org/ audiogames/slenderlostvision/, last access: Feb. 2017.

${ }^{4}$ Slender - The Eight Pages: https://de.wikipedia.org/ wiki/Slender_Man, last access: Feb. 2017.

${ }^{5}$ Adventure at C: http: //www.vgstorm.com/aac/, last access: Feb. 2017.

${ }^{6}$ Simon: https://en.wikipedia.org/wiki/Simon (game), last access: Feb. 2017
}

\subsubsection{Board games}

Many board games have been adapted for the visually impaired. In those games, all relevant information is announced by the computer, e.g., the name of the player who is to move next, the card placed, the cards owned by the player or the diced numbers. Such adaptations also make it easier for impaired and unimpaired people to play board games together.

An example of an audio board games is "Blind Gamers Hearts". It is an adaptation of the card game "Hearts" where a computer voice announces the active player and his placed card. The player then uses the arrow keys to scroll through his cards, each card being read aloud. The desired card is placed with the Enter key and the game continues.

\subsection{Navigation in virtual audio environments}

Lokki et al. [3] studied auditory navigation in virtual acoustic environments without visual feedback. They set up an experiment, where the users would navigate to multiple sound sources placed in a virtual 2D world with keyboard commands to walk forward, backwards and to turn left or right. They examined the effect of different factors, including:

- Panning methods: interaural time difference (ITD), ITD + interaural level difference (ILD) and ITD + head-related transfer function (HRTF)

- Simulated acoustic environments: direct sound only, direct sound + reflections and direct sound + reflections + artificial reverb

Lokki et al. counted how often the users found the target area and measured the time spent in the navigation task. In their experiments, the users performed best with ITD + ILD panning. The best acoustic environment for the navigation task was direct sound only, while direct sound + reflections + artificial reverb performed worst [3]. Since we wanted our game to sound aesthetically pleasing and since the differences measured by Lokki et al. are only moderate, we chose to use reflections and artificial reverberation nevertheless. Also the navigation task in the study by Lokki et al. was limited to targets located within a single room so that the reflections did not convey information relevant to the navigation task. In a more complex (and for a computer game more typical) setup with multiple connected rooms and spaces, reflections could be helpful since the user could perceive the spatial layout, e.g., by perceiving a lack of reflections from an area where a passage leads to another room. In a further study Gröhn, Lokki and Takala examined auditory and audio-visual navigation in a 3D space, where the user could move freely in all six degrees of freedom [4]. Interestingly, the users improved when they received auditive cues in addition to visual cues.

Picinali et al. created a system that lets visually impaired people explore buildings using virtual acoustics so that they get along better when they visit the building in real life [5]. The users wore headphones with an orientation sensor, which was used in combination with a joystick to navigate. Virtual sound sources were placed in the room, e.g., a group of men talking. Furthermore, the user could generate sounds (finger snapping, footsteps) to explore the sound properties of the virtual room. Picinali et al. showed that their users were able to create relatively accurate mental maps

\footnotetext{
${ }^{7}$ Blind Gamers Hearts: http://www.omninet.net.au/ irhumph/bghearts.htm, last access: Feb. 2017.
} 
of the building [5]. Our users (with unimpaired sight) were not able to construct such accurate mental maps. This is consistent with a study that compared sighted and visually impaired people in a comparable audio navigation task [6]. Of course, other factors such as the differences in interaction (orientation sensor and joystick vs. mouse and keyboard) or differences in the spatial sound system could also have contributed to the different outcome.

But audio navigation in an audio-only computer game does not have to rely on real-world acoustics. Completely new mappings could be used, e.g., those proposed and examined by Lorenz and Schuster [7]. They modulated various parameters of a synthesizer (including pitch, tempo, LFO, reverberation, panning and timbre) by the distance between the mouse cursor and the target. They measured how long it took for the users to acquire the target and the distance that the mouse cursor traveled. They also computed the directional accuracy, which is the mean deviation of the angle between the optimal and the real direction of mouse movement. Based on these measurements, Lorenz and Schuster conclude that tempo, pitch and timbre performs best while "stereo panning [...] seem[s] hardly suitable for navigation tasks" [7]. Of course their navigation task was clearly different than ours. Their sonification techniques could however be applied for navigation in first person adventure games. Of course, in that case the game would not sound realistic any more.

\section{SIMULATION OF ROOM ACOUSTICS}

Our game is a first-person adventure. For such games we deem the quality of spatial sound reproduction to be crucial. This is even more important than in traditional video games, since the sound is the only modality. Sounds of a virtual world can be converted synthetically into binaural signals [8]. Such approaches depend, on the one hand, on the physical behavior of the sound waves, and on the other hand, on the biological presuppositions and functions of human hearing. An in-depth introduction to physical and physiological foundations can be found in $[9,10,11,12]$.

In 1999, Mueller and Ullmann conducted experiments in which reflections were simulated in a three-dimensional space [13]. The simulation was based on the idea of ray tracing, a common technique in 3D rendering. Beams emanating from a sound source are emitted. When a collision with an object such as a wall occurs, a further sound source is produced, which in turn emits further beams. This operation is performed until the specified maximum number of beams is reached. Beams that collide with the virtual representation of the listener are used to calculate the final sound. In addition, the angle of incidence or the exact location of the collision determines how the HRTF filter is calculated for the respective beam. The computers used at that time required about 13 hours for the calculation of a ten-second sample with a limitation to 10,000 virtual sound sources [13]. Today, ray tracing simulation of room acoustics is possible in real-time, e.g., by making use of the GPU [14]. Alternatively, the propagation of sound waves can be simulated. This is physically more correct but computationally more expensive. Again the GPU can be used for an efficient implementation [15].

The most common, commercially available programming frameworks for spatial audio are Aureal A3D, Creative EAX, FMOD, Microsoft DirectSound 3D, and OpenAL. These can be divided into two groups:

- The frameworks in the first group simulate a space without paying attention to the exact geometry of the space to which the simulation is applied by using prefabricated presets, e.g., with fixed impulse responses. Therefore, the calculation of the audio signals does not react dynamically to the movement of the listener in the virtual space. Such frameworks include DirectSound 3D, Creative EAX and OpenAL.

- The frameworks in the other group calculate sound reflections dynamically in real time and can therefore produce sounds that depend on the position of the listener in the virtual space. This group is mainly represented by Aureal A3D [16, p. 49] and dearVR [17]. Other systems have not been commercialized or have not achieved a certain level of commercial use. Since Aureal A3D has been discontinued, we chose dearVR as spatial audio framework for our audio game.

dearVR uses the ray tracing to determine early reflections. This is based on the actual geometry of the room in which the sound event takes place. dearVR considers only those reflections, which reach the listener after a single contact with a wall or any other object. The direct sound and the early reflections are then spatialized using a HRTF. The phase cancellations resulting from the delay in the summation of the signals are similar to those occurring in reality enough to give the player an impression of an actual three-dimensional space.

All further reflections are not calculated in this way but are produced with a reverberation effect. This reverb can be selected from a list of prefabricated presets. This reverb is calculated independently of the position and orientation of the listener in the virtual space. The consequence of this is that the localization of a sound source is made more difficult, especially if the reverb is too loud in relation with the direct sound and the reflections. We used relatively soft reverb in our game.

\section{OVERVIEW OF THE GAME}

\subsection{Story}

The game "Fire in Neptune" is based on the following story: The protagonist Steffen Vogel regularly visits the casino "Neptune". One night, he loses a considerable sum of money, he gets drunk and wakes up at home the next morning. During that night, the casino did burn down completely. Steffen is arrested and questioned for suspicions of arson. The recordings of the surveillance cameras in the casino have been destroyed. However, the surveillance camera of an adjacent building shows Steffen leaving the casino and driving away hurriedly before the fire breaks out. Nobody else survived the inferno.

He says time and again that he is innocent, but he cannot remember the exact course of the evening. He declares himself willing to support his statement by the "interactive memory protocol" (IMP). This is a device that measures the brain currents and provides access to lost memories. As a result, the previous evening can be reconstructed. This is where the first scene of the game begins. Steffen is instructed in the functioning of the IMP and travels virtually into the past to live through the evening.

In the game, the player has the opportunity to move freely. The first chapter takes place in the casino. Here, the player can play with the game machine or at the roulette table. In addition, he can get drinks at the bar, listen to the songs at the jukebox or listen to the conversation of other casino visitors. 


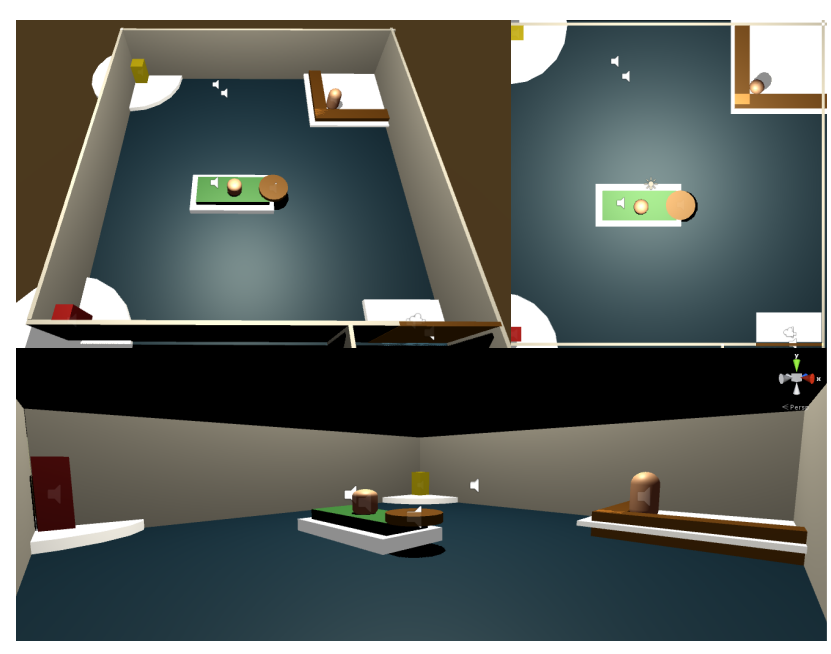

Figure 1: The layout of the casino. The 3D environment was used for development only and is not shown to the users of the audio game.

\subsection{Game control}

The player can move freely in the virtual space using the mouse to control the viewing direction and the keys "W, A, S, D" to move forward, left, back, and right. This is a widely game control interface, which is used in many first-person shooter and adventure games. If a question is put to the protagonist Steffen, the player can affirm with the "E" key or negate by pressing the "Q" key. We limited the freedom of the viewing direction to the horizontal pane. This is different from games with similar controls, where you can also look up and down. This makes it easier to localize sounds.

Fig. 1 shows the casino. The user enters and leaves the casino through the door at the lower right. The roulette table is located in the center of the room, the bar at the top left corner, the jukebox at the top right corner, and the playing machine at the bottom right corner. The $3 \mathrm{D}$ environment was used for development only and is not shown to the users of the audio game. The game was developed using the Unity game engine for 3D modeling and game logic. We used dearVR to create spatial audio (see Section 3.2).

\section{OBSERVATIONS}

We employed an iterative design process. Six persons were regularly observed during the development of the game. The test persons differed greatly in their experience in playing computer games. All participants were without visual impairment.

The players were confronted with the game while we observed the interactions taking place and any specific issues that arose. In doing so, no help or background information was provided to the players. In order to understand the player's actions in the virtual world, we duplicated the audio signal and listened with our own headphones. Because we had an exact idea of the structure and architecture of the rooms, we were able to "see" exactly where the player was at any time. So we could, e.g., perceive the differences between the desired, planned behavior of the player and his or her actual actions. That way we were able to identify many problems ourselves. The players often reported difficulties later on.

\subsection{Orientation at Sound Sources}

Each of the test players showed the same behavior at this point: When placed in a room where only one audible signal was heard, the player oriented and walked toward it. If at a certain point in the game, there is nothing to be heard, then there is no guiding point to which the player can orient himself/herself or to determine how far he has turned in which direction. In this case, the players responded in a confused or uncertain way. Even if the sound is threatening and players move away from it, the orientation and positioning in the virtual space is always related to a sound.

In practice this means that the game developer must place a sound source at every location the player is supposed to move to. At the tutorial in the beginning of the game, the player hears a voice giving him/her the instruction to go through the door behind the voice in the wall. As soon as this sentence is pronounced, the voice is silenced and the player has to go through a door. The door is marked with an earcon that plays continuously. This sound is modeled as a soft, pulsing sound. The sound continues to play until the player has reached the door and the next sequence is initiated.

In 2002, Andersen tried out a similar approach [18], marking exits in a virtual room with distinct earcons. Unfortunately, the players were not able to target the exits. We did not face these problems in our game. We presume this could be due to the fact that the audio signal in Andersens game was limited to stereo without HRTFs, so signals from the front or from behind would sound identical.

Andersen realized that the player did not have to hear the exit but the space behind it [18]. Thus, dampened sound sources from the room behind the exit could be heard when the player passed the opening to the other room. Additionally, this solution is less artificial than using earcons and may therefore provide a better immersion. Also the player is already prepared for what he will experience in the next room.

In general, the natural sound of an object should be preferred. However, there are always situations in which the object in question does not sound in reality. Then the game developer does not have another option than to associate an artificial sound [19].

\subsection{No absolute spatial orientation}

For a sighted person, it can be difficult to imagine an unknown space without the help of his/her eyes. This was observed in all the players and many verbalized this fact. In most cases, the players moved from one audible object to the other without being able to make a precise statement about their absolute position in the room. For example, the player could say, "When I go to the jukebox, I'll first move to the bar." But not: "The jukebox is in the opposite corner of the room." See Fig. 2.

Some players were completely disorientated and unable to assess whether two objects are directly adjacent or very far apart. Others could at least remember the routes that they traversed. We also observed that after some practice, users moved more efficiently and in a more goal-oriented manner (see Section 6).

\subsection{Collisions with Obstacles}

To orient oneself in a dark room, one does not rely exclusively on hearing but also on proprioceptive and tactile senses. It is, for example, common to advance in the dark with extended arms in order to avoid or mitigate collisions with walls or other obstacles. 

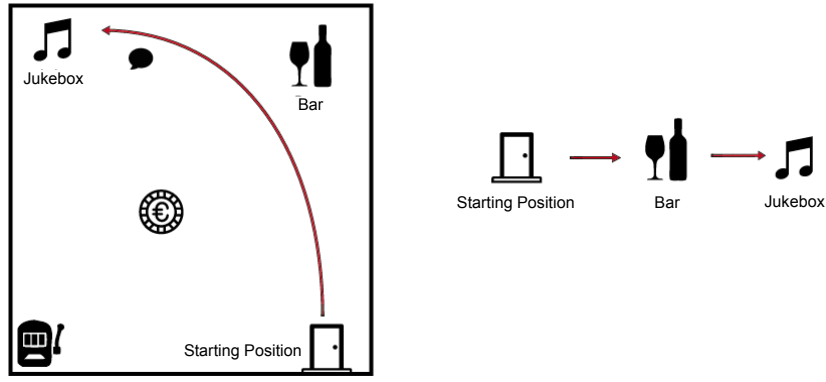

Figure 2: Left the actual route, right the perception of the player.

Lacking those modalities, collisions must be communicated acoustically in audio games.

The game "Slender - Lost Vision" (see Section 2.1.3) solves this problem with a knock sound that plays when the protagonist collides with an object. Unfortunately, the knocking sound is played as a mono sound. The knocking thus indicates that the player has encountered an obstacle but does not make any statement as to the angle at which the collision occurred. Therefore, there is no information available to the player on the basis of which he/she could opt for a course correction to the right or left.

Andersen went a step further. The signal used to indicate the contact with the wall uses stereo to distinguish whether the collision with a wall took place frontally or at an angle [18]. In our game, a knock is heard when the player hits an obstacle. In contrast to "Slender Lost Vision" and similar to Andersens game, this knocking can be heard from the direction in which the collision occurred.

We observed that some test users avoided to leave the immediate proximity of the wall and thus did not walk to the middle of the room. An almost empty space provokes this behavior much more than one in which many sound sources can be heard. Objects placed in free space are generally harder to find than those located along a wall. A possibility to break that "behavioral pattern" is to place disturbances along the room's walls.

In our game we placed these objects adjacent to a wall, which should be discovered quickly. The elements, which the player should only explore at a later stage of the game, were placed in the middle of the room.

\section{EVALUATION AND DISCUSSION}

\subsection{Method}

The players received no information or instructions on the game. However, if a player got stuck, he or she could ask for help.

The game begins with a short tutorial, in which the player is instructed how to control the game. Then, in the next section, the background story of the game is introduced. After the intro, the player is located in the casino and is confronted with an uncommented soundscape. From this point on the player takes full control. After finishing the first chapter of the game, the players rated 13 statements about their impression of the game on a 6 point Likert scale and made a sketch of the floor plan from memory.

Subsequently, the players entered the virtual casino a second time and got the instruction to move to the game machine, the bar and the jukebox in that order. The time required was recorded for each of the stations. When the player arrived at the jukebox, the

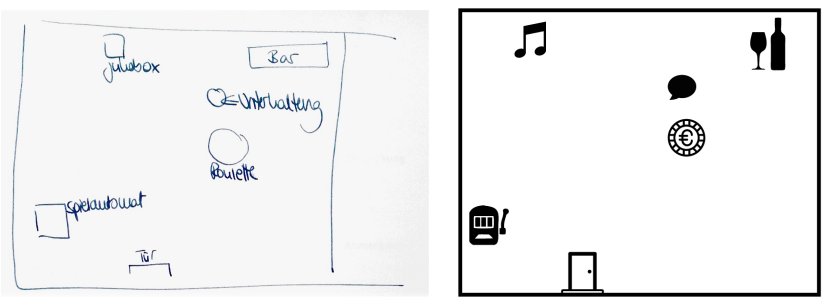

Figure 3: On the left the original plan drawn by the player, on the right the coded version.

final position of the route, the player was taken back to the starting position. The players then ran the same route a second time, then a third time while the time was being measured again. After that, the players were given the opportunity to correct the first sketch and make a second memory sketch. They were asked to describe in their own words what they liked most about the game and what could be improved.

The evaluation was conducted by ten test players and lasted about 35 minutes per player. The age of the players is between 18 and 28 years, all had unimpaired sight. Three of the players said they were inexperienced in dealing with ego perspective games. The memory sketches, which the players made, were coded, as shown in Fig. 3. If necessary, the drawing was rotated so that the door was placed at the lower edge. In this way the individual sketches could be compared with each other better than in their original form.

\subsection{Results}

\subsubsection{Game Play}

The players spent an average of 13 minutes in the game. 18\% of the time was spent in the tutorial, $16 \%$ in the intro, $47 \%$ in the casino and $19 \%$ in the final navigation test, which also took place in the casino.

Firstly, it is noticeable that all 10 players state that it was a new experience for them to play an audio game (6). They liked the game (5.2) and they would gladly continue to play it (5.6). They found the story to be interesting (5.5) and the game concept to be easy to grasp (5.4). When audio games are discussed in literature, visually impaired players are often stated as the primary target group. Since this group is comparatively small, it is understandable that big game companies produce only few audio games. In 2007, blind players expressed their dissatisfaction with this situation [1]. Our questionnaire shows that players with healthy sight can find audio games interesting. Perhaps the target group for audio games is larger than generally assumed.

The players did not always know what they had to do (3.8). The individual players disagreed with each other with values ranging from 2 to 5. Probably this is a question of the playing style: While some players stopped and waited for new instructions or something to happen, others continued to explore the environment and came into action independently.

Both in positive and negative criticism, the players noticed different aspects. One player pointed out that at first it was difficult for him to distinguish between a sound source in front of and behind him. This is consistent with the use of generic HRTFs. This problem could be solved by a personlized HRTF, such as proposed 


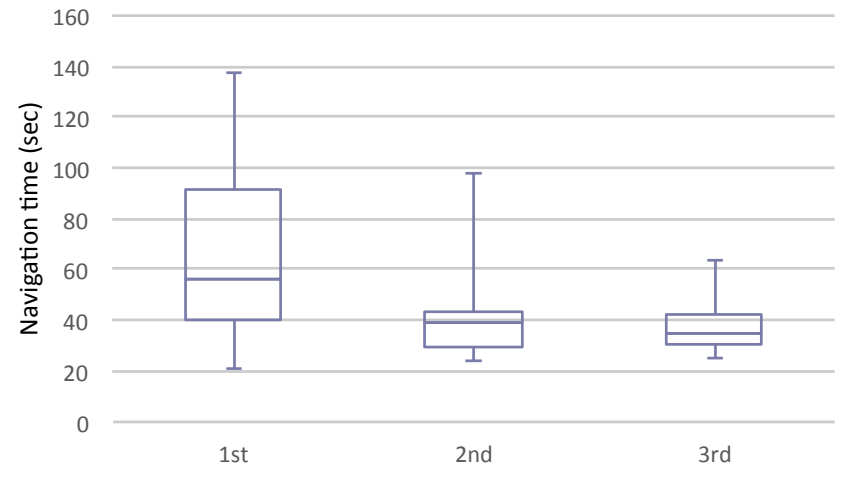

Figure 4: The time required to complete the 1st, 2nd and 3rd pass through the casino. The box plots show the minimum, the $25 \%$ quantile, the median, the $75 \%$ quantile and the maximum.

in [20].

The players took pleasure in the dialogues and the possibility to control the course of the game. In addition, the control was described as "simple, but appropriate" and the principle of navigating through hearing was described as a "new experience" and "very interesting". In addition, the the "beautiful" or "memorable sounds / voices" and the story of the game were also mentioned positively. One player liked that the scene is created in the imagination of the player, making it individual and interesting.

\subsubsection{Orientation and Navigation}

The players said the voices were easy to understand (6.0) and the sounds were easy to recognize (5.2). In addition, the control was described as intuitive (5.1). Although they were able to determine relatively well from which direction a sound came (4.2), they report that navigation in the room was not that easy (3.5) and that they could not imagine the space well (3.2).

There was a clear difference between the players with and those without experience in ego perspective games. While the experienced players used mouse and keyboard, the inexperienced players navigated almost exclusively with the keyboard. This resulted in a motion pattern composed of straight lines and 90 degree angles. While these players also estimated their own perception of the space as inaccurate (3.3) and felt even more unsure about their position (3.0) than the other players (3.7), their memory sketches were much closer to the actual layout of the room.

We wanted to measure how navigation time changes with playing experience. In fact, the average time required to complete the given route (from the door to the game machine, then to the bar and finally to the jukebox) reduced to $63 \%$ respectively $57 \%$ of the initial amount in the second and third run. Figure 4 provides an overview of the measured times.

We assumed that the user got more efficient in navigating with game experiences. The average time to complete the path decrease from the first time they traversed the path $(67.3 \mathrm{~s})$, to the second (42.4 s) and the third time (38.2 s). To examine statistical significance, we used one-way ANOVA with post-hoc Tukey Honest Significance (HSD) Test. The ANOVA results show that the time differs significantly between the three rounds $(p=0.043)$. The posthoc Tukey HSD test did however not reach a significance level of $p<0.05$ for any pairwise comparison. When comparing between the first and third round, significance was almost achieved with $p=0.052$. Probably, significance would eventually be reached with more participants.

\section{LESSONS LEARNED}

From our experiences we want to formulate two design ideas:

- Use a sufficient number of unique sound sources.

- Use a simple floor plan.

Sufficient number of unique sound sources: As already stated, the players usually localize a sound source, orient themselves and then move towards in a more or less straight line. Once the player has reached the source, there has to be at least one additional meaningful sound source otherwise an acoustic dead end has been reached. Also, the sound sources should be unique. In an early version of the game, we had 12 virtual loudspeakers installed on the ceiling of the casino playing the same music. Since the sound signals were the same, the virtual loudspeaker setup did not help to navigate. Therefore, we replaced the loudspeakers with a jukebox located in one corner of the virtual room. As Andersen points out, the sounds of footsteps could be used to give the player more information about the room [18], e.g., when walking over a carpet or over stone.

Simple floor plan: The orientation can also be simplified by keeping the architecture simple [18]. It seems that many players assume that the playing area is rectangular. As a game developer, this assumption can be used in two ways. On the one hand, the space can be intentionally created in such a way that the player's expectations are met. On the other hand, it may be appropriate to deliberately make navigation more challenging. In such cases, the space may be L-shaped, octagonal or star-shaped.

\section{REFERENCES}

[1] R. van Tol and S. Huiberts, "What blind gamers want the industry to know. compiled for the 2006 game developers convention," 2006, last access: Feb. 2017. [Online]. Available: http://captivatingsound.com/ what-blind-gamers-want-the-industry-to-know/

[2] M. J. Wolf, "Genre and the video game," in The medium of the video game, M. J. Wolf and R. H. Baer, Eds. Austin: University of Texas Press, 2001, pp. 113-134.

[3] T. Lokki, M. Grohn, L. Savioja, and T. Takala, "A case study of auditory navigation in virtual acoustic environments," in International Conference on Auditory Display (ICAD), 2000, pp. $145-150$.

[4] M. Gröhn, T. Lokki, and T. Takala, "Comparison of auditory, visual, and audiovisual navigation in a 3D space," $A C M$ Trans. Appl. Percept., vol. 2, no. 4, pp. 564-570, Oct. 2005.

[5] L. Picinali, A. Afonso, M. Denis, and B. F. Katz, "Exploration of architectural spaces by blind people using auditory virtual reality for the construction of spatial knowledge," International Journal of Human-Computer Studies, vol. 72, no. 4, pp. 393-407, 2014.

[6] A. Afonso, A. Blum, B. F. Katz, P. Tarroux, G. Borst, and M. Denis, "Structural properties of spatial representations in 
blind people: Scanning images constructed from haptic exploration or from locomotion in a 3-d audio virtual environment," Memory \& Cognition, vol. 38, no. 5, pp. 591-604, 2010.

[7] R. H. Lorenz and H. Schuster, "Auditory pointers," in Works in Audio and Music Technology, A. Berndt, Ed. Dresden, Germany: TUDpress, 2015, pp. 1-30.

[8] J. Engdahl, "An evaluation of 3D sound APIs," Bachelor Thesis, University of Karlstad, Sweden, 2002.

[9] R. Lerch, G. M. Sessler, and D. Wolf, Technische Akustik: Grundlagen und Anwendungen. Springer-Verlag, 2009.

[10] P. Meier, "Studioakustik," in Handbuch der Audiotechnik, S. Weinzierl, Ed. Springer Science \& Business Media, 2008, pp. 267-311.

[11] W. Ahnert and H.-P. Tennhardt, "Raumakustik," in Handbuch der Audiotechnik, S. Weinzierl, Ed. Springer Science \& Business Media, 2008, pp. 181-266.

[12] J. Blauert and J. Braasch, "Räumliches Hören," in Handbuch der Audiotechnik, S. Weinzierl, Ed. Springer Science \& Business Media, 2008, pp. 87-121.

[13] W. Mueller and F. Ullmann, "A scalable system for 3D audio ray tracing," in IEEE International Conference on Multimedia Computing and Systems, vol. 2. IEEE, 1999, pp. 819-823.

[14] N. Röber, U. Kaminski, and M. Masuch, "Ray acoustics using computer graphics technology," in 10th International Conference on Digital Audio Effects (DAFx-07), 2007, pp. 117-124.

[15] N. Röber, M. Spindler, and M. Masuch, "Waveguide-based room acoustics through graphics hardware," in International Computer Music Conference (ICMC), 2006, pp. 21-28.

[16] J. Dvorak, C. Pirillo, and W. Taylor, Online!: The Book. Prentice Hall Professional, 2004.

[17] dearVR, "dearVR 3D audio reality engine - user manual v1.1,” 2016, last access: Feb. 2017. [Online]. Available: http: //www.dear-reality.com/support/dearVR_user_manual.pdf

[18] G. Andersen, "Playing by ear: Using audio to create blind-accessible games," 2002, last access: Feb. 2017. [Online]. Available: http://www.gamasutra.com/resource_ guide/20020520/andersen_pfv.htm

[19] M. A. Oren, C. Harding, and T. Bonebright, "Evaluation of spatial abilities within a $2 \mathrm{~d}$ auditory platform game," in Proceedings of the 10th international ACM SIGACCESS conference on Computers and accessibility. ACM, 2008, pp. 235-236.

[20] A. Meshram, R. Mehra, H. Yang, E. Dunn, J.-M. Franm, and D. Manocha, "P-HRTF: efficient personalized HRTF computation for high-fidelity spatial sound," in Mixed and Augmented Reality (ISMAR), 2014 IEEE International Symposium on. IEEE, 2014, pp. 53-61. 